\title{
3-D Analysis of Nanomaterials using Electron Tomography
}

\author{
P.A.Midgley, M. Weyland, R. Dunin-Borkowski, L. Laffont, J.M. Thomas and T. Yates
}

Department of Materials Science and Metallurgy, University of Cambridge, Pembroke Street, Cambridge, CB2 3QZ UK

As the nanotechnology revolution gathers pace there will be an increasingly strong need for imaging techniques that allow a high spatial resolution view of nanostructures and nanodevices. By their nature such objects will be three-dimensional and any imaging technique must allow for this. Such complex structures at the nanoscale have been the subject of interest for life scientists for decades and these have been imaged in three dimensions since the late 1960's using electron tomography [1]. With physical science specimens beginning to approach the complexity of some biological specimens it is very appropriate that we turn to electron tomography to reveal the three-dimensional structure of nanoscale devices.

Tomography is a two-stage process as illustrated schematically in Fig. 1 [2]. Firstly a series of images (projections) are recorded of the object at successive tilts. The image contrast must satisfy the 'projection criterion' that says, in essence, that the recorded signal must be monotonically related to the projected thickness. For life sciences, BF images in which mass-thickness contrast dominates is suitable, but in the physical sciences, where in general, crystalline objects will produce Fresnel and Bragg contrast, BF (coherent) imaging is wholly unsuitable. In general, we need to turn to an incoherent form of imaging in which the signal is insensitive to the diffraction conditions and which satisfies the projection criterion. There are a number of signals that can be detected in an electron microscope that will lead to an incoherent image. The two that have been developed so far are high angle annular dark field (HAADF) imaging using a STEM approach [3] and energy filtered TEM in which core-loss images can be considered incoherent under certain circumstances $[4,5]$. The second part of the process is the reconstruction itself, performed using a 'back-projection' routine. Each image (projection) is back-projected along the original tilt angle into a three dimensional space. The overlap of all projections then leads to the reconstruction of the 3D object. Each projection can also be thought as a central slice through Fourier space and thus the back-projection is equivalent to a 3D inverse transform of these Fourier slices. For computational efficiency the back-projection method is preferred. The reconstruction process is fraught with problems and artefacts can dominate unless care is taken. It is vital that as many images as possible are recorded over as large a range of tilt as possible. The angular sampling determines the $3 \mathrm{D}$ resolution but this is worsened if the tilt range is limited. A range of $+/-40^{\circ}$ will lead to an elongation of each object parallel to the optic axis by a factor of 3 . For $+/-70^{\circ}$ this is reduced to only $30 \%$ and for most purposes this modest loss of resolution is not a problem. We have further limited artefacts by employing an iterative backprojection routine that constrains the final reconstruction to match the original projection.

Fig. 2 shows results from a 3D tomographic reconstruction of a magnetite nanocrystal found in the 'backbone' of a magneto-tactic bacterium. The original images were recorded using STEM HAADF and thus were predominantly insensitive to diffraction conditions. The faceting of the crystals is well revealed in the reconstruction. Fig. 3(a) shows a perspective view of a reconstruction (from STEM HAADF images) of a heterogeneous catalyst composed of bi-metallic Pd-Ru particles supported on an MCM-41 mesoporous silica framework. The image in Fig. 3(b) shows the box indicated in (a) rotated to look down the 6-fold axis of the MCM-41 to reveal $3 \mathrm{~nm}$ diameter channels and a single sub- $\mathrm{nm} \mathrm{Pd}_{6} \mathrm{Ru}_{6}$ nanoparticle anchored to the inside wall [6]. 


\section{References}

[1] R.A. Crowther, D. J. de Rosier and A. Klug, Proc. Roy. Soc Lond. A 317 (1970) 319.

[2] A.J. Koster et al., J. Phys. Chem. B, 104 (2000) 9368-9370.

[3] P.A. Midgley et al., Chem. Comm. 18 (2001) 907.

[4] G. Mobus and B.J. Inkson, Applied Physics Letters 79 (9) (2001) 1369.

[5] M. Weyland and P.A. Midgley, Microsc. Microanal (2003), in press.

[6] This research was supported by the EPSRC and Royal Commission for the Exhibition of 1851.

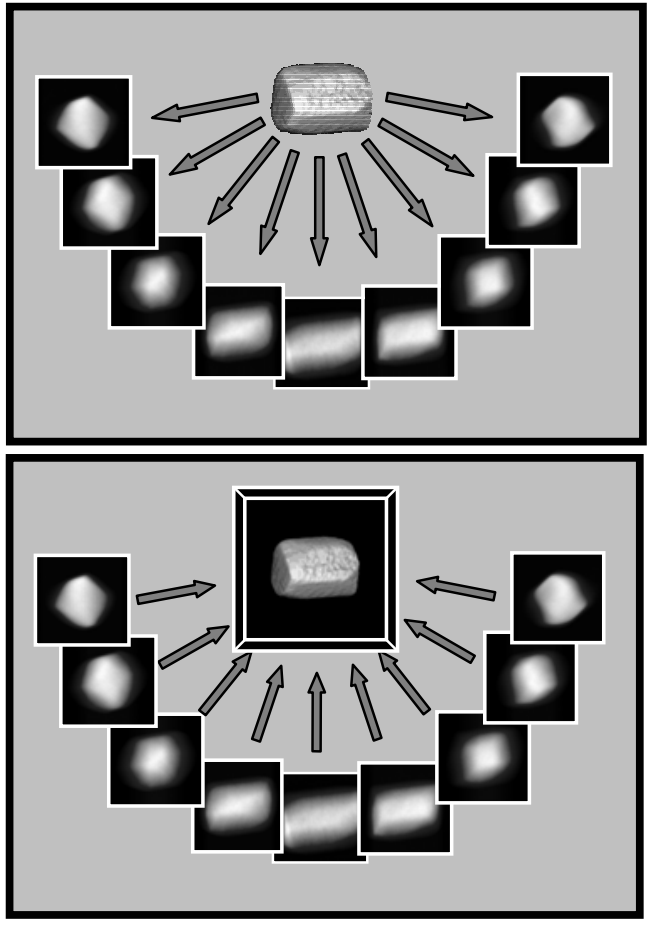

FIG. 1. A schematic diagram to illustrate the tomography process. A series of images (projections) are recorded at different tilts from a 3D object. These images are then back-projected to reconstruct the 3D object. (After [2].)

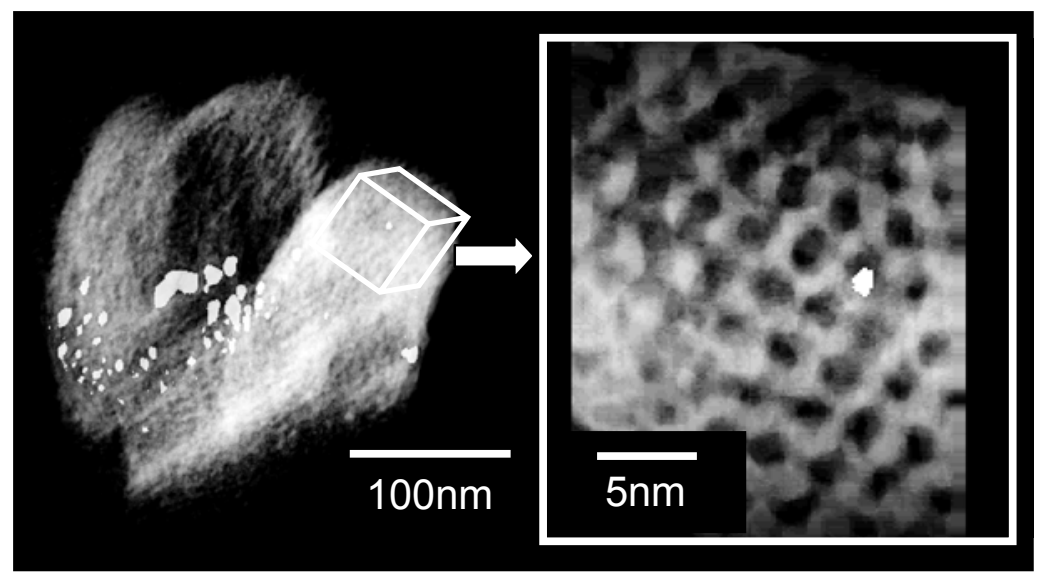

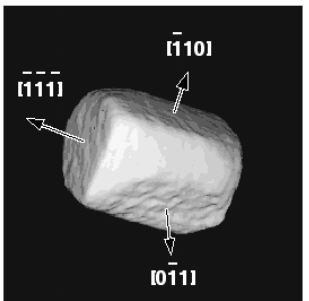
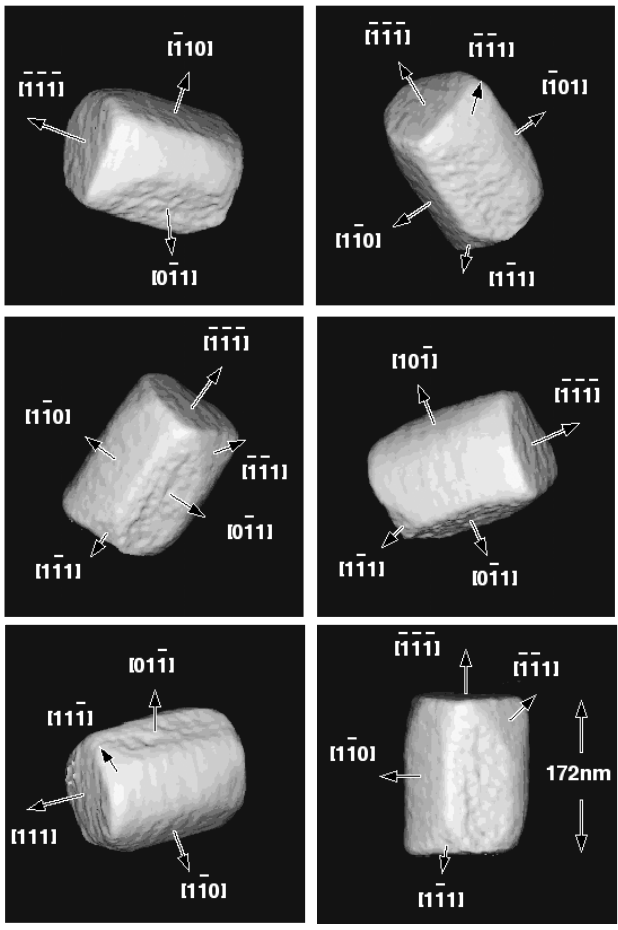

FIG. 2. A montage of perspective views of a magnetite nanocrystal found in the backbone of a magneto-tactic bacterium. The faceting is revealed well in the surface rendered object.

FIG. 3. (a) A voxel projection of a tomographic reconstruction of a heterogeneous catalyst composed of Pd-Ru nanoparticles supported within a mesoporous silica (MCM-41) support. The original STEM HAADF images enable the Z-contrast to be revealed in this reconstruction, the particles appearing far more intense than the silica support.(b) The box indicated in (a) has been rotated to view down the 6-fold axis of the MCM-41 to reveal $3 \mathrm{~nm}$ diameter channels and a single $\mathrm{Pd}_{6} \mathrm{Ru}_{6}$ nanoparticle anchored to the wall. 